\title{
OBSERVAÇÕES SOBRE O COMPORTAMENTO DE LUTZOMYIA WHITMANI,ANTUNES \& COUTINHO, 1939 (DIPTERA, PSYCHODIDAE) EM CONDIÇÕES NATURAIS, NA CIDADE DE GOIÂNIA-GOIÁS
}

Heloisa Aparecida Machado Naves * Maria Elisa Santos Dourado Carvalho**

\section{RESUMO}

Coletaram-se 168 exemplares de $\mathrm{Lu}$. whitmani em capturas realizadas com isca humana em solo e copa de árvore, isca animal e armadilhas luminosas (Shannon e CDC) nos horários matutino, vespertino e noturno, na Chácara Naves, nos anos de 1987,1988,1991 e 1992.

O maior número de exemplares foi obtido no ano de 1988, seguindo-se 1987,1992 e 1991.

Sua maior incidência ocorreu nos meses de agosto, setembro e julho.

Isca humana no solo foi a preferida por esta espécie $(51,19 \%)$; seguindo-se armadilha de Shannon $(41,66 \%)$; isca animal e armadilha CDC $(2,97 \%)$ e isca humana em plataforma $(1,19 \%)$.

Com relação à frequência horária observou-se uma maior ocorrência do flebótomo entre 20 e 21 horas $(32,14 \%)$ dos exemplares.

UNITERMOS: Lutzomyia whitmani, iscas, armadilhas, freqüência mensal e horária

\section{INTRODUÇÃO}

.. Profa. do Depto. de Parasitologia.IPTSP/UFG Goiânia-Goiás

- Bióloga do Depto. de Parasitologia-IPTSP-UFG C.P. 131 Goiânia-Goiás

Recebido para publicação em 04/08/94 
NAVES,H.A.M. \& CARVALHO,M.E.S.D. Observaçðes sobre o Comportamento de Lutzomyia whitmani, Antunes \& Coutinho, 1939 (Diptera,Psychodidae) em Condiçð̄es Naturais, na Cidade de Goiânia-Goiás. Rev.Pat.Trop.,23 ( 2 ): 235-242.jul./dez. 1994

Evidências da importância de Lu. whitmani como transmissora de leishmaniose tegumentar foram obtidas em estudos pioneiros de PESSOA \& COUTINHO (1941) que detectaram nesta espécie infecção natural por promastigotas de Leishmania braziliensis.

Observaçðes de ordem epidemiológica entre as quais alta densidade, freqüuencia aos domićlios e antropofilia conduziram à suspeita da transmissão de leishmaniose tegumentar por Lu.whitmani segundo BARRETO (1943).

CUBA et al. (1982), observaram após sete dias de infecção, formas promastigotas ativas ao nível de faringe de flebótomos alimentados em patas de hamsters com L.braziliensis braziliensis.

VEXENAT et al. (1986) infectaram Lu.whitmani em lesões leischmanióticas de cães parasitados por

L.b.braziliensis na região endêmica de Três Braços, Bahia.

Em Goiás, MARTINS et al. (1962) registraram Lu.whitmani nos municípios de Anápolis,Rianópolis e Sítio d'Abadia; COELHO et al. (1965), em Mineiros, coletaram com isca humana 141 exemplares.

Em municípios goianos essa espécie prodominou no período de 1984-1985, segundo LUSTOSA et al, (1986).

A ocorrência de maior número de casos de leishmaniose em Goiás coincidiu com regið̋es onde houve desmatamento para instalação de fazendas agropastoris abertura de estradas, sendo Lu.whitmani segunda espécie em número nestas localidades conforme CARVALHO et al. (1989).

Em Goiânia, NAVES et al. (1992) registraram pela primeira vez a ocorrência desta espécie.

\section{MATERIAIS E MÉTODOS}

A área estudada localiza-se na região norte de Goiânia próxima ao Bairro Fama, Setor Meia Ponte e Vila Itatiaia. A estação de coleta situa-se em mata de Interflúvio, que se caracteriza pela queda não uniforme das folhas, principalmente nos períodos mais secos do ano. Devido à queda de folhas, ramos, flores, frutos, cascas e troncos, o solo fica recoberto o que é importante na reciclagem de nutrientes.

A margem da mata, encontram-se casas permanentemente habitadas, piscinas e abrigos de animais.

Realizaram-se 79 capturas nos anos de 1987,1988,1991 e 1992, totalizando 766 horas de coleta.

Para observar a preferência alimentar quanto a hospedeiros utilizou-s- de canídeo (Canis), galináceo (Gallus) e suíno (Scrofa). Com relação à isca humana as
NAVES,H.A.M. \& CARVALHO,M.E.S.D. Observaçðes sobre o Comportamento de Lutzomyia whitman Antunes \& Coutinho, 1939 (Diptera,Psychodidae) em Condiç̃̃es Naturais, na Cidade de Goiânia-Goiás. Rev.Pat.Trop.,23 (2) : 235-242.jul./dez. 1994

coletas foram realizadas por duas pessoas que no solo e em plataforma funcionvam como isca e ao mesmo tempo capturador.

Com relação à armadilhas luminosas utilizou-se de Shannon em mata, $\mathrm{CDC}$ em mata, galinheiro, pocilga e plataforma.

\section{RESULTADOS E DISCUSSÃO}

Obtiveram-se 168 exemplares de Lutzomyia whitmani na Chácara Naves, Goiânia-Goiás, nos anos de 1987, 1988, 1991 e 1992. (TABELA 1).

Em três municípios do Estado de Goiás com suspeita de leishmaniose tegumentar, LUSTOSA et al. (1986) registraram 111 exemplares de Lu.whitmani nos anos de 1984 e 1985. Em publicação de 1989 CARVALHO et al. (1989) relatam a ocorrência de 36 espécimes em nove municipios goianos, sem no entanto estudarem a fauna flebotomínica de nossa capital.

Em Campo Grande, Rio de Janeiro, onde foram constatados vários casos humanos e caninos de L.T.A LIMA et al. (1981) obtiveram exemplares desta espécie no período de maio a junho do referido ano.

Em Goiânia nos quatro anos estudados registrou-se maior ocorrência de Lu.whitmani no ano de 1988 (48,80\% dos exemplares); 1987 (25,00\%): 1992 (20,83\%) e $1991(5,35 \%)$. (TABELA 1$)$.

TABELA 1. Numero mensal de Lu.whitmani coletado em Goiânia-Goiás, nos anos de 1987,1988,1991 e 1992.

\begin{tabular}{lccccccccccccc}
\hline Anos & & \multicolumn{1}{c}{} & \multicolumn{1}{c}{ Meses } & & & & & Total \\
& Jan & Fev & Mar & Abr & Mai & Jun & Jul & Ago & Set & Out & Nov & Dez \\
\hline 1987 & - & - & - & - & - & - & - & 03 & 30 & 05 & 04 & - & 42 \\
1988 & - & 04 & 08 & - & - & - & - & 70 & - & - & - & - & 82 \\
1991 & - & - & - & - & - & - & 09 & - & - & - & - & - & 09 \\
1992 & 03 & - & - & - & 04 & 04 & 13 & 03 & 04 & 02 & 02 & - & 35 \\
TOTAL & 03 & 04 & 08 & - & 04 & 04 & 22 & 76 & 34 & 07 & 06 & - & 168 \\
\hline
\end{tabular}


NAVES,H.A.M. \& CARVALHO,M.E.S.D. Observaçðes sobre o Comportamento de Lutzomyia whitmani, Antunes \& Coutinho, 1939 (Diptera,Psychodidae) em Condiçð̃es Naturais, na Cidade de Goiânia-Goiás. Rev.Pat.Trop.,23 (2): 235-242.jul./dez. 1994.

Observou-se uma maior incidência desta espécie no mês de agosto $(45,23 \%)$, seguindo-se setembro(20,23\%); julho (13,09\%); março $(4,76 \%)$; outubro $(4,16 \%)$; novembro (3,57\%); fevereiro, maio e junho $(2,38 \%)$ e janeiro $(1,78 \%)$. (TABELA 2).

As coletas nos meses de abril e dezembro foram negativas para $\mathrm{Lu}$.whitmani.

TABELA 2. Número mensal de Lu.whitmani coletado simultaneamente em isca humana ao nivel do solo e emplataforma, isca animal,armadilhas de Shannon e CDC, nos anos de 1987, 1988, 1991 e 1992.

\begin{tabular}{|c|c|c|c|c|c|c|c|c|c|c|c|c|c|}
\hline \multirow{2}{*}{$\begin{array}{l}\text { Iscas e } \\
\text { armadilhas }\end{array}$} & \multicolumn{12}{|c|}{ Meses } & \multirow[t]{2}{*}{ Total } \\
\hline & Jan & Fev & Mar & Abr & Mai & Jun & Jul & Ago & Set & Out & Nov & Dez & \\
\hline $\begin{array}{l}\text { Isca Humana } \\
\text { (solo) }\end{array}$ & 03 & - & 04 & - & 04 & 04 & 15 & 32 & 19 & 03 & 02 & - & 86 \\
\hline Isca Humana & - & 02 & - & - & - & - & - & - & - & - & - & - & 02 \\
\hline $\begin{array}{l}\text { (plataforma) } \\
\text { Isca animal }\end{array}$ & - & - & - & - & - & - & - & 03 & - & - & 02 & - & 05 \\
\hline Shannon & - & 02 & 04 & - & - & - & 07 & 39 & 15 & 01 & 02 & - & 70 \\
\hline $\mathrm{CDC}$ & - & - & - & - & - & - & - & 02 & - & 03 & - & - & 05 \\
\hline TOTAL & 03 & 04 & 08 & - & 04 & 04 & 22 & 76 & 34 & 07 & 06 & - & 168 \\
\hline
\end{tabular}

Segundo FORATTINI (1954), há espécies de flebótomos mais ou menos resistentes às mudanças climáticas durante o decorrer das estações do ano. Algumas são encontradas durante os meses mais frios e secos; outras desaparecem durante tais épocas. Existem também vários fatores que influenciam na incidência dos mesmos em um mesmo local, ainda que sob as mesmas condições de temperatura e umidade.

Com relação às iscas e armadilhas utilizadas, verificou-se maior eficácia para isca humana no solo ( $51,19 \%$ dos exemplares) sendo a mesma atuante em todos os meses do ano exceto fevereiro, abril e dezembro.

Em agosto obteve-se $37,20 \%$ dos exemplares coletados com esta isca, nos anos estudados.

Registrou-se Lu. whitmani em plataforma apenas no mês de fevereiro.
NAVES,H.A.M. \& CARVALHO,M.E.S.D. Observaçð̃es sobre o Comportamento de Lutzomyia whitmani, Antunes \& Coutinho, 1939 (Diptera,Psychodidae) em Condiçø̃es Naturais, na Cidade de Goiânia-Goiás. Rev.Pat.Trop.,23 (2):235-242.jul./dez. 1994.

Em isca animal nos meses de agosto e novembro foram coletados $2.97 \%$ do total geral de exemplares.

Armadilha de Shannon contribuiu com $41,66 \%$ dos flebótomos obtidos. Foi mais produtiva em agosto, setembro e julho.

Foram capturados em armadilha CDC, $2,97 \%$ do total geral registrado. Os poucos exemplares ocorreram nos meses de agosto e outubro. (TABELA 2).

AGUIAR et al. (1989), em foco ativo de leishmaniose tegumentar no norte do estado do Paraná, usando de isca humana, armadilhas de Shannon e Falcão observaram em periodomicílio, margem da floresta e floresta maior eficácia de armadilha de Shannon nos três ambientes estudados. Em Goiás CARVALHO et al. (1989) coletaram através desta armadilha $87,73 \%$ dos flebótomos coletados em 11 municípios.

Com relação à frequência horária, observou-se um crescente aumento do número de exemplares a partir do crepúsculo vespertino. Os horários mais produtivo foram: $20-21$ horas $(32,14 \%$ dos flebótomos); $19-20$ horas $(27,97 \%)$ e $18-19$ horas (22,61\%). (TABELA 3).

TABELA 3. Frequência horária de Lu. whitmani coletado em Goiânia, nos anos de 1987,1988,1991 e 1992.

\begin{tabular}{|c|c|c|c|c|c|c|c|c|c|c|c|c|c|}
\hline Horário & & & & & & Yese & & & & & & & Total \\
\hline & Jan & Fev & Mar & $\mathrm{Abr}$ & Mai & Jun & Jul & Ago & Set & Out & Nov & Dez & \\
\hline $09-10$ & - & - & - & - & - & 02 & - & - & - & - & - & - & 02 \\
\hline $10-11$ & - & - & - & - & - & - & - & - & - & - & - & - & - \\
\hline $11-12$ & - & - & - & - & - & - & - & - & - & - & - & - & - \\
\hline $12-13$ & - & - & - & - & - & - & - & - & - & - & - & - & - \\
\hline $13-14$ & - & - & - & - & - & - & - & - & - & - & - & - & - \\
\hline $14-15$ & - & - & - & - & - & - & - & - & - & - & - & - & - \\
\hline $15-16$ & - & - & - & - & - & - & - & 02 & - & - & - & - & 02 \\
\hline $16-17$ & - & - & - & - & 01 & - & 03 & 04 & - & - & - & - & 08 \\
\hline $17-18$ & - & - & - & - & - & - & 07 & 09 & 01 & - & - & - & 17 \\
\hline
\end{tabular}


NAVES,H.A.M. \& CARVALHO,M.E.S.D. Observaçðes sobre o Comportamento de Lutzomyia whitmani, Antunes \& Coutinho, 1939 (Diptera,Psychodidae) em Condiçðes Naturais, na Cidade de Goiânia-Goiás. Rev.Pat.Trop.,23 (2): 235-242.jul./dez. 1994.

\begin{tabular}{cccccccccccccc}
$18-19$ & 02 & 02 & 02 & - & 03 & 01 & 06 & 16 & 03 & 02 & 01 & - & 38 \\
$19-20$ & - & 02 & 03 & - & - & 01 & 05 & 20 & 12 & 02 & 02 & - & 47 \\
$20-21$ & 01 & - & 03 & - & - & - & 01 & 25 & 18 & 03 & 03 & - & 54 \\
TOTAL & 03 & 04 & 08 & - & 04 & 04 & 22 & 76 & 34 & 07 & 06 & - & 168 \\
\hline
\end{tabular}

AGUIAR et al. (1985), no Parque Nacional da Serra dos Órgãos, Rio de Janeiro, observaram maior ocorrência de flebotomíneos entre 17-19 horas. Em Campo Grande, no mesmo Estado LIMA et al. (1981) observaram discreta predominância do número de flebótomos coletados no período de 16:20 às 20:45h em relação ao período de 8-11h. Em nosso trabalho o período da manhã contribuiu com apenas dois exemplares de Lu.whitmani.

\section{SUMMARY}

Observations on behaviour of Lutzomyia whitmani,Antunes \& Coutinho, 1939

(Diptera, Psychodidae) in natural conditions, in Goiânia city, Goiás.

168 exemplaries of $\mathrm{Lu}$. whitmani were collected in captures realized with human baits in ground and on top of tree, animal bait, and luminous traps (Shannon and CDC), in periods: morning, afternoon and night, in Naves Farm, in years 1987, $1988,1991,1992$.

The major occurrence was in August, September and July.

This type prefered human bait in ground $(51,19 \%)$, then Shannon's trap $(41,66 \%)$, animal bait and CDC trap $(2,97 \%)$, and human bait in platform $(1,19 \%)$.

Related to the time frequency, it was observed a major occurence of flebotomus between 08:00pm and 09:00pm, 32,14\% of the exemplaries.

KEYWORDS: Lutzomyia whitmani, baits, traps, month and time frequency.

\section{REFERÊNCIAS BIBLIOGRÁFICAS}

01. AGUIAR, G. M.; SCHUBACK,P.D'A.; VILELA,M.L \& AZEVEDO,A.C.R.de. Aspectos da ecologia dos flebótomos do Parque Nacional da Serra dos Órgãos,
NAVES,H.A.M. \& CARVALHO,M.ES.D. Observaçes sobre o Comportamento de LuEomyi Rev Pat Rev.Pat.Trop.,23 ( 2 ) : 235-242.jul./dez. 1994

Rio de Janeiro, II. Distribuição vertical. (Diptera, Psychodidae, Phlebotominae). Mem.Inst.Oswaldo Cruz,80, (2): 194-197, 1985.

02. AGUIAR, G. M.de.; VILELA,M.L.; FERREIRA,V.A. \& SANTOS,T.G. dos. Ecologia dos flobótomos em um recente foco ativo de Leishmaniose tegumentar no norte do estado do Paraná (Diptera,Psychodidae,Phlebotominae). Mem.Inst. Oswaldo Cruz, 84, (4 supl.): 7-8, 1989.

03. BARRETO, M.P. Observações sobre a biologia, em condiçø̃es naturais, dos flebótomos do Estado de São Paulo (Diptera,Psychodidae). São Paulo, 1943 (Tese - Faculdade de Medicina da Universidade de São Paulo.)

04. CARVALHO,M.E.S.D.; LUSTOSA, E.deS. \& NAVES,H.A.M. Contribuição ao conhecimento da fauna flebotomínica do estado de Goiás e Distrito Federal.II 1986-1987. Rev.Pat.Trop. 18 (1):7-14, 1989.

05. COELHO,M.V.; CUNHA,A.S. \& FALCÃO,A.R. Notas sobre um foco de Calazar no sudoeste do estado de Goiás. Rev.Brasil.Malariol. Doenças Trop.,17: 143$148,1965$.

06. CUBA, C. A.; VEXENAT, J. A.; LlanOS, C. A.; MARSDEN, P. D.; BARRETO,A.C. \& ROSA, A. de C. Experimental infections of wild caugth specimens of Lutzomyia (N.) whitmani (Diptera, Psychodidae) and their use for Leishmania identification. IX Reunião Anual de Pesquisa Básica em Doenças de Chagas. Caxambu, Minas Gerais, p.149. 1982.

07. FORATTINI,O.P. Algumas observações sobre a biologia de flebótomos (Diptera,Psychodidae) em região da bacia do rio Paraná (Brasil). Arqv. Fac.Hig.Saúde Públ. 8: 15-136, 1954.

08. LIMA, L.C.R.de.; MARZOCHI, M. C. de A. \& SABROZA,P.C. Flebotomíneos em área de ocorrência de Leishmaniose tegumentar no bairro de Campo Grande, Rio de Janeiro. Brasil. Rev.Brasil.Malariol. D. Trop. 33: 64-74, 1981.

09. LUSTOSA, E. de S.L; NAVES,H.A.M.; CARVALHO,M.E.S.D. \& BARBOSA,W Contribuição ao conhecimento da fauna flebotomínica do Estado de Goiás. 1984-1985. Nota prévia I. Rev.Pat.Trop.15 (1): 7-11-, 1986. 
NAVES,H.A.M. \& CARVALHO,M.E.S.D. Observaçð̌es sobre o Comportamento de Lutzomyia whitmani, Antunes \& Coutinho, 1939 (Diptera,Psychodidae) em Condiçð̌es Naturais, na Cidade de Goiânia-Goiás. Rev.Pat.Trop.,23 (2): 235-242.jul./dez. 1994.

10. MARTINS,A.V.; FALCÃO,A.L. \& SILVA,J.E. Nota sobre os flebótomos do Estado de Goiás com a descriçào de duas espécies novas e da fềmea de Lutzomyia longipennis (Barreto, 1946) e a redescrição do macho de L. evandroi (Costa Lima e Antunes, 1936). (Diptera,Psychodidae). Rev.Brasil.Malariol. Doença Trop. $14: 379-394,1962$.

11 - NAVES,H.A.M.; CARVALHO,M.E.S.D. \& LUSTOSA, E.de S. Ocorrência de $L u$. whitmani (Antunes \& Coutinho, 1939) (Diptera,Psychodidae) em GoiâniaGoiás. In: SEMINÁRIO EM SAÚDE PÚBLICA,1., 1992., Goiânia. Rev.Pat.Trop. 21 (1,Supl.): 33, 1992.

12. PESSOA, S. B. \& COUTINHO,J.O. Infecção natural e experimental do flebótomos pela Leishmania braziliensis no estado de São Paulo. Hospital.20: 25-35, 1941.

13. VEXENAT, J. A.; BARRETO, A. C. \& ROSA, A. de C. Infecção experimental de Lutzomyia whitmani em cães infectados com Leishmania braziliensis braziliensis. Mem.Inst. Oswaldo Cruz, 81 (1): 125-126, 1986. 